\title{
Infeções Urinárias Causadas por Enterobacteriaceae Produtoras de $\beta$-Lactamases de Espetro Expandido Adquiridas na Comunidade num Hospital de Nível III: Um Estudo Retrospetivo
}

\author{
Urinary Tract Infections Caused by Community-Acquired \\ Extended-Spectrum $\beta$-Lactamase-Producing \\ Enterobacteriaceae in a Level III Hospital: A \\ Retrospective Study
}

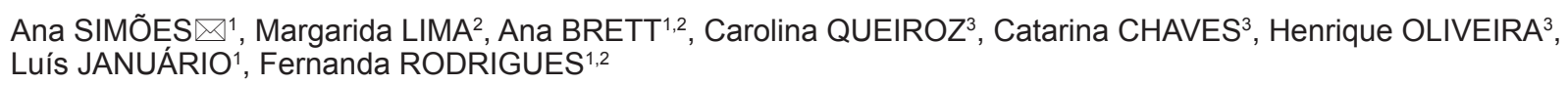

Acta Med Port 2020 Jul-Aug;33(7-8):466-474 - https://doi.org/10.20344/amp.12338

RESUMO

Introdução: A emergência de bactérias produtoras de $\beta$-lactamases de espetro expandido é um problema mundial, com importância crescente nas infeções adquiridas na comunidade, nomeadamente nas infeções urinárias. Os dados pediátricos de utilização de antimicrobianos não carbapenemos nestas infeções são escassos. O objetivo do estudo foi analisar a terapêutica antibiótica instituída nas infeções urinárias causadas por estes agentes, assim como a evolução clínica e laboratorial.

Material e Métodos: Estudo retrospetivo efetuado num hospital pediátrico entre junho de 2007 e dezembro de 2017. Foram incluídas todas as crianças com urocultura positiva para Enterobacteriaceae produtoras de $\beta$-lactamases.

Resultados: Foram diagnosticadas 175 infeções urinárias causadas por Enterobacteriaceae produtoras de $\beta$-lactamases, das quais 34 (19\%) foram adquiridas na comunidade: 25 Escherichia coli (74\%), 4 Klebsiella pneumoniae (12\%), 4 Proteus mirabilis (12\%) e 1 Proteus vulgaris (3\%). Em 30 (88\%) episódios tratou-se da primeira infeção urinária. Após conhecimento do microrganismo e suas suscetibilidades, $33(97 \%)$ crianças foram reavaliadas e $24(71 \%)$ repetiram urocultura, que foi positiva em três (13\%). Em seis (18\%) casos foi alterado o antimicrobiano. No mês subsequente, quatro (12\%) crianças tiveram nova infeção urinária e 30 (88\%) crianças realizaram investigação imagiológica, sem deteção de malformações nefro-urológicas.

Discussão: Na última década, cerca de $20 \%$ das infeções urinárias causadas por Enterobacteriaceae produtoras de $\beta$-lactamases foram adquiridas na comunidade, com um número relativamente estável ao longo dos anos. Estas crianças não apresentavam malformações nefro-urológicas.

Conclusão: Embora o número de casos seja pequeno, a evolução clínica e microbiológica mostrou que a maioria foi tratada com sucesso com antimicrobianos não carbapenemos, com baixa ocorrência de novos episódios.

Palavras-chave: Antibacterianos; $\beta$-lactamases; Criança; Enterobacteriaceae; Infecções Comunitárias Adquiridas; Infecções por Enterobacteriaceae; Infecções Urinárias

\section{ABSTRACT}

Introduction: The emergence of $\beta$-lactamases producing bacteria is a problem worldwide, with increasing importance in communityacquired infections, especially in urinary tract infections. Data regarding the use of non-carbapenem antimicrobials in these infections are scarce. The aim of this study was to analyse the treatment and outcome of urinary tract infections caused by community-acquired $\beta$-lactamase-producing bacteria in children.

Material and Methods: Retrospective study performed in a level III paediatric hospital, between June 2007 and December 2017. All children with $\beta$-lactamase-producing Enterobacteriaceae identified in aseptically collected urine culture were included.

Results: A total of 175 urinary infections caused by $\beta$-lactamases producing bacteria were diagnosed, 34 (19\%) were community-acquired: 25 Escherichia coli (74\%), 4 Klebsiella pneumoniae (12\%), 4 Proteus mirabilis (12\%) and 1 Proteus vulgaris (3\%). In 30 (88\%) cases, it was the first urinary infection. After identification of the microorganism and antimicrobial susceptibility, $33(97 \%)$ children were re-evaluated and $24(71 \%)$ had a repeat urine culture, which was positive in three $(13 \%)$. In six (18\%) cases, antibiotic treatment was modified. Four (12\%) children had another UTI in the following month. In 30 (88\%) children, imaging was carried out, with no nephrourological malformations detected.

Discussion: In the last decade, about $20 \%$ of urinary infections caused by $\beta$-lactamase-producing Enterobacteriaceae were community-acquired with a relatively stable number of cases over the years. No nephro-urological malformations were identified in these children.

Conclusion: Although the number of cases is small, the clinical and microbiological outcomes showed that most were successfully treated with non-carbapenem antibiotics, with low recurrence of new episodes of urinary tract infections.

Keywords: Anti-Bacterial Agents; beta-Lactamases; Child; Community-Acquired Infections; Enterobacteriaceae; Enterobacteriaceae Infections; Urinary Tract Infections

1. Serviço de Urgência e Unidade de Infeciologia. Hospital Pediátrico. Centro Hospitalar e Universitário de Coimbra. Coimbra. Portugal.

2. Faculdade de Medicina. Universidade de Coimbra. Coimbra. Portugal.

3. Serviço de Patologia Clínica. Centro Hospitalar e Universitário de Coimbra. Coimbra. Portugal.

$\bowtie$ Autor correspondente: Ana Simões. anasofiapediatria@outlook.pt

Recebido: 18 de maio de 2019 - Aceite:04 de novembro de 2019 | Copyright $\odot$ Ordem dos Médicos 2020 


\section{INTRODUÇÃO}

A infeção urinária (IU) é uma patologia muito comum em idade pediátrica. A sua prevalência global em crianças febris é de $7 \%$, variando, no entanto, com a idade, raça/ etnia e sexo. ${ }^{1} \mathrm{O}$ seu tratamento adequado é essencial, nomeadamente para prevenir a formação de cicatrizes renais, com consequente atingimento da função renal. ${ }^{2}$ As bactérias envolvidas pertencem maioritariamente à família das Enterobacteriaceae: Escherichia coli (E. coli) é responsável por $60 \%$ a $90 \%$ dos casos, seguindo-se Klebsiella pneumoniae (K. pneumoniae) e Proteus mirabilis ( $P$. mirabilis). ${ }^{3}$

As $\beta$-lactamases de espetro expandido (ESBL) são enzimas que hidrolizam o anel $\beta$-lactâmico presente em várias classes de antibióticos, inativando-o, conferindo desta forma às bactérias que as produzem resistência à penicilina, às cefalosporinas de primeira, segunda e terceira gerações e ao aztreonam. Podem ainda ser resistentes a aminoglicosídeos, fluoroquinolonas e cotrimoxazol. São suscetíveis aos carbapenemos ${ }^{4} \mathrm{e}$, apesar de apresentarem frequentemente suscetibilidade in vitro à associação amoxicilina/ ácido clavulânico, a sua utilização no tratamento destas infeções é ainda controversa. ${ }^{5}$

A identificação das primeiras estirpes de Enterobacteriaceae produtoras de ESBL ocorreu na Alemanha em 1983, estando inicialmente limitada a infeções nosocomiais em doentes adultos em unidades de cuidados intensivos. ${ }^{6}$ Os primeiros casos adquiridos na comunidade (AC) foram reportados em $2000^{7}$ e as primeiras descrições em crianças surgiram entre 1999 e 2003 em França, inicialmente como causa de doença invasiva em unidades de cuidados intensivos neonatais e pediátricos. ${ }^{8,9}$

Atualmente estas bactérias são um problema à escala global, com importância crescente nas infeções AC, nomeadamente nas IU e intra-abdominais, ${ }^{10}$ sendo uma causa emergente de IU em crianças. Este fenómeno verifica-se principalmente na Ásia, onde cerca de $40 \%$ das Enterobacteriaceae uropatogénicas são produtoras de ESBL. ${ }^{11}$ Mas, apesar de serem mais frequentes em países em desenvolvimento, têm também aumentado em países desenvolvidos ${ }^{12}$ tal como os EUA, onde a prevalência de ESBL detetadas em crianças, englobando infeções AC e associadas aos cuidados de saúde, passou de $0,26 \%$ em 2001 para $0,92 \%$ em $2010-2011 .{ }^{13}$ O mesmo tem ocorrido em alguns países europeus, de que são exemplo a França e a Alemanha. ${ }^{12,14,15}$

Para além de maior tempo de internamento e de custos mais elevados quando comparados com os atribuídos a IU causadas por outros microrganismos, ${ }^{16}$ foi também reportado que, em casos de IU com bacteriémia, a mortalidade foi superior, particularmente em recém-nascidos. ${ }^{17,18}$

Os carbapenemos são os antibióticos indicados para o tratamento destas infeções. No entanto, há já um número crescente e muito preocupante de Enterobacteriaceae produtoras de carbapenemases, responsáveis por uma mortalidade elevada, ${ }^{19}$ pelo que é do maior interesse reduzir o seu uso.

Antimicrobianos não carbapenemos têm sido utilizados com sucesso no tratamento destas IU em adultos, não se demonstrando diferenças nas taxas de cura clínica e bacteriológica, na mortalidade aos 14 dias e na recorrência destas infeções, comparativamente aos doentes tratados com carbapenemos..$^{20-24}$

Sendo escassa a informação sobre a utilização de antibióticos não carbapenemos no tratamento de IU causadas por bactérias produtoras de ESBL em pediatria, pretendemos avaliar a terapêutica instituída, a evolução clínica (resolução dos sintomas nomeadamente febre e sintomatologia urinária) e laboratorial (resultado da urocultura de controlo) e a recorrência de IU causadas por estas bactérias AC.

\section{MATERIAL E MÉTODOS \\ Estudo populacional retrospetivo}

Trata-se de um estudo retrospetivo, descritivo, sem intervenção, efetuado no Hospital Pediátrico - Centro Hospitalar e Universitário de Coimbra (CHUC), um hospital de nível III que presta cuidados de saúde a crianças e jovens até aos 17 anos e 364 dias, na região centro do país, abrangendo 392112 crianças e adolescentes dos zero aos 18 anos (Instituto Nacional de Estatística, 2017). No período do estudo ocorreram, em média, 58550 observações/ano no Serviço de Urgência. Até 2011, a faixa etária abrangida era até aos 13 anos e em fevereiro desse ano foi alargada até aos 18 anos. Os dados microbiológicos foram obtidos a partir da base de dados do Serviço de Patologia Clínica do CHUC. Incluíram-se todas as crianças com idades compreendidas entre os zero e os 18 anos, com isolamento de bacilo Gram negativo produtores de ESBL na urina, entre junho de 2007 (data em que teve início a identificação laboratorial destes microrganismos) e dezembro de 2017 (10,5 anos).

O estudo foi aprovado pela Comissão de Ética para a Saúde do Centro Hospitalar e Universitário de Coimbra.

\section{Métodos microbiológicos}

Os bacilos Gram negativos foram isolados da urina através de cultura, e a identificação e suscetibilidades antibióticas das Enterobacteriaceae foram realizadas recorrendo ao sistema automatizado Vitek-2 (bioMérieux, Marcy L'Etoile, France) e aos métodos E-teste e combinação de discos.

As concentrações inibitórias mínimas foram interpretadas de acordo com os valores de referência do European Committee on Antimicrobial Susceptibility Testing (EUCAST).

\section{Critérios de inclusão}

Consideraram-se infeções AC as que ocorreram em doentes que não apresentavam fatores de risco e nos quais o isolamento da bactéria na urina ocorreu nas primeiras 48 horas de internamento.

Definimos cura clínica como a resolução dos sintomas, nomeadamente febre e/ou sintomatologia urinária (disúria, 
polaquiúria, dor lombar); definimos cura bacteriológica a existência de melhoria nos parâmetros da análise sumária de urina (redução do número de leucócitos por campo, ausência de nitritos e de bactérias em urina fresca) e urocultura de controlo negativa. Perante a identificação de uma Enterobacteriaceae produtora de ESBL todas a crianças fizeram controlo laboratorial às 48 - 72 horas após início de tratamento antibiótico.

Foram considerados fatores de risco: existência de internamento prévio, intervenção cirúrgica, uso de antibióticos ou presença de dispositivo médico nos 30 dias anteriores ao episódio, realização de terapêutica renal de substituição, uso de profilaxia para IU, colonização gastrointestinal por Enterobacteriaceae produtora de ESBL, ventilação invasiva ou corticoterapia prolongada nos últimos 12 meses, baixo peso ao nascimento e existência de malformações nefro-urológicas e/ou infeções urinárias recorrentes. , 25-28 $^{2}$

Só foram incluídas colheitas assépticas de urina, por punção suprapúbica, cateterismo vesical ou jato médio após lavagem.

\section{Informação recolhida}

A partir da base de dados do Serviço de Patologia Clínica do CHUC foi obtida informação sobre a estirpe identificada em cada caso e as suas suscetibilidades aos antibióticos, sendo assim identificadas as crianças a incluir no estudo.

Foi recolhida a seguinte informação através da consulta dos processos clínicos: idade, sexo, data da colheita de urina, método de colheita, diagnóstico final (cistite ou pielonefrite), presença de fatores de risco, terapêutica empírica instituída, alteração do antibiótico, necessidade de internamento, realização de urocultura de controlo e seus resultados assim como a realização de exames imagiológicos e seus resultados, repetição de IU nos seis meses subsequentes.

As definições de pielonefrite aguda e cistite aguda foram baseadas no protocolo de atuação clínica local.

Foi considerada pielonefrite aguda toda a IU com ou sem febre em criança de idade inferior a dois anos ou IU com ou sem febre com dor lombar associada em qualquer idade. A cistite aguda foi definida como IU não febril em criança com idade igual ou superior a dois anos. Foi definida como IU recorrente a ocorrência de dois pielonefrites agudas ou de três ou mais cistites agudas, ou uma pielonefrite aguda associada a uma cistite aguda.

Nas crianças com idade inferior a 24 meses, as manifestações clínicas podem ser inespecíficas, sendo a febre o principal sinal. Após os 24 meses, a presença de febre e/ou dor lombar orienta para uma localização do processo inflamatório no parênquima renal (pielonefrite aguda). Sintomatologia urinária como disúria, polaquiúria ou incontinência

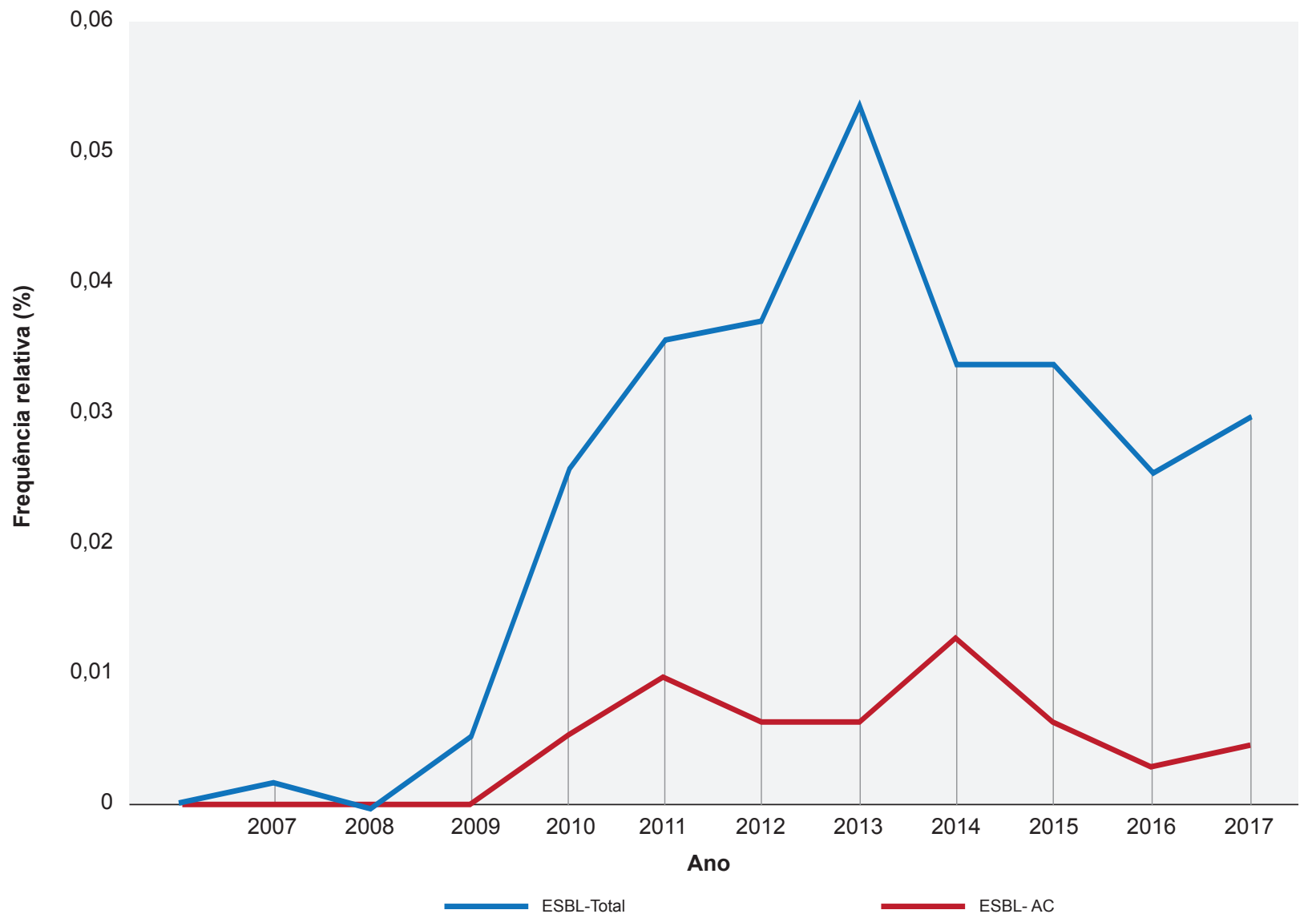

Figura 1 - Frequência relativa (\%) das infeções urinárias causadas por bactérias produtoras de $\beta$-lactamases e adquiridas na comunidade, no período de 2007 a 2017 
orientam para uma localização no aparelho urinário baixo (cistite aguda). ${ }^{29}$

O diagnóstico de IU em crianças baseia-se na realização do teste rápido de urina (nitritos e leucócitos) ou sumária de urina II (nitritos, leucócitos e bactérias) e da urocultura. $^{1}$

O diagnóstico é confirmado por urocultura positiva por colheita assética (punção vesical suprapúbica positiva se $\geq$ $10^{3}$ bactérias $/ \mathrm{mL}$, cateterismo vesical positivo se $\geq 10^{4}$ bactérias $/ \mathrm{mL}$ ou jato médio positivo se $\geq 10^{5}$ bactérias $/ \mathrm{mL}$ ). ${ }^{29,30}$

Os dados foram registados e processados pelo programa Microsoft Office Excel $2016^{\circledR}$. Foi realizada uma análise estatística descritiva (a função mediana nas variáveis quantitativas e a frequência relativa nas variáveis nominais). Foram utlizados gráficos descritivos (gráficos de barras 2D, gráficos de linhas 2D) e descrições tabulares para ilustrar os resultados.

Foi utilizado um gráfico de linhas que representa a frequência relativa ao longo do tempo das IU causadas por bactérias produtoras de ESBL de forma a ilustrar as alterações verificadas entre os anos 2007 e 2017; um histograma de frequência para representar a frequência das IU causadas por bactérias produtoras de ESBL-AC distribuída por coortes de idades; e um gráfico de barras para representar a percentagem de bactérias produtoras de ESBL resistentes aos antibióticos testados.

\section{RESULTADOS}

Frequência relativa de IU causadas por bactérias produtoras de ESBL-AC e evolução ao longo do período do estudo

Durante o período do estudo foram diagnosticadas 175 IU causadas por bactérias produtoras de ESBL, das quais
34 (19\%) foram AC. A sua frequência relativa ao longo do tempo é apresentada na Fig. 1.

Destaca-se um aumento mantido do número total de IU causadas por bactérias produtoras de ESBL até 2013 (ano em que foi atingido o valor máximo), mantendo-se relativamente estável desde então. Em relação às IU causadas por estas bactérias AC, as primeiras foram diagnosticadas em 2010 e, apesar de alguma variabilidade, os números mantiveram-se baixos e estáveis ao longo dos anos, com uma mediana de quatro casos/ano (máximo de oito casos em 2014 e mínimo de dois casos em 2016).

\section{Características demográficas e clínicas dos casos de IU causadas por bactérias produtoras de ESBL-AC}

Pertenciam ao sexo feminino 18 (53\%) casos. A idade mediana foi de dois anos e cinco meses (1 mês - 16 anos). Em 30 (88\%) casos tratou-se da primeira IU. A distribuição por frequência de idades é apresentada na Fig. 2.

A colheita de urina foi realizada por jato médio em 27 (79\%) casos, por cateterismo vesical em seis (18\%) e por punção suprapúbica em um (3\%). Todas as colheitas foram realizadas no Serviço de Urgência.

O diagnóstico foi de pielonefrite aguda em 19 (56\%) casos e cistite em 15 (44\%).

\section{Dados microbiológicos das IU causadas por bactérias produtoras de ESBL-AC}

As bactérias identificadas foram E. coli em 25 (74\%), K. pneumoniae em quatro (12\%), P. mirabilis também em quatro $(12 \%)$ e $P$. vulgaris em um $(3 \%)$.

Na Fig. 3 são apresentadas as resistências das bactérias isoladas a diferentes antibióticos. Todas demonstraram suscetibilidade in vitro aos carbapenemos (meropenem/

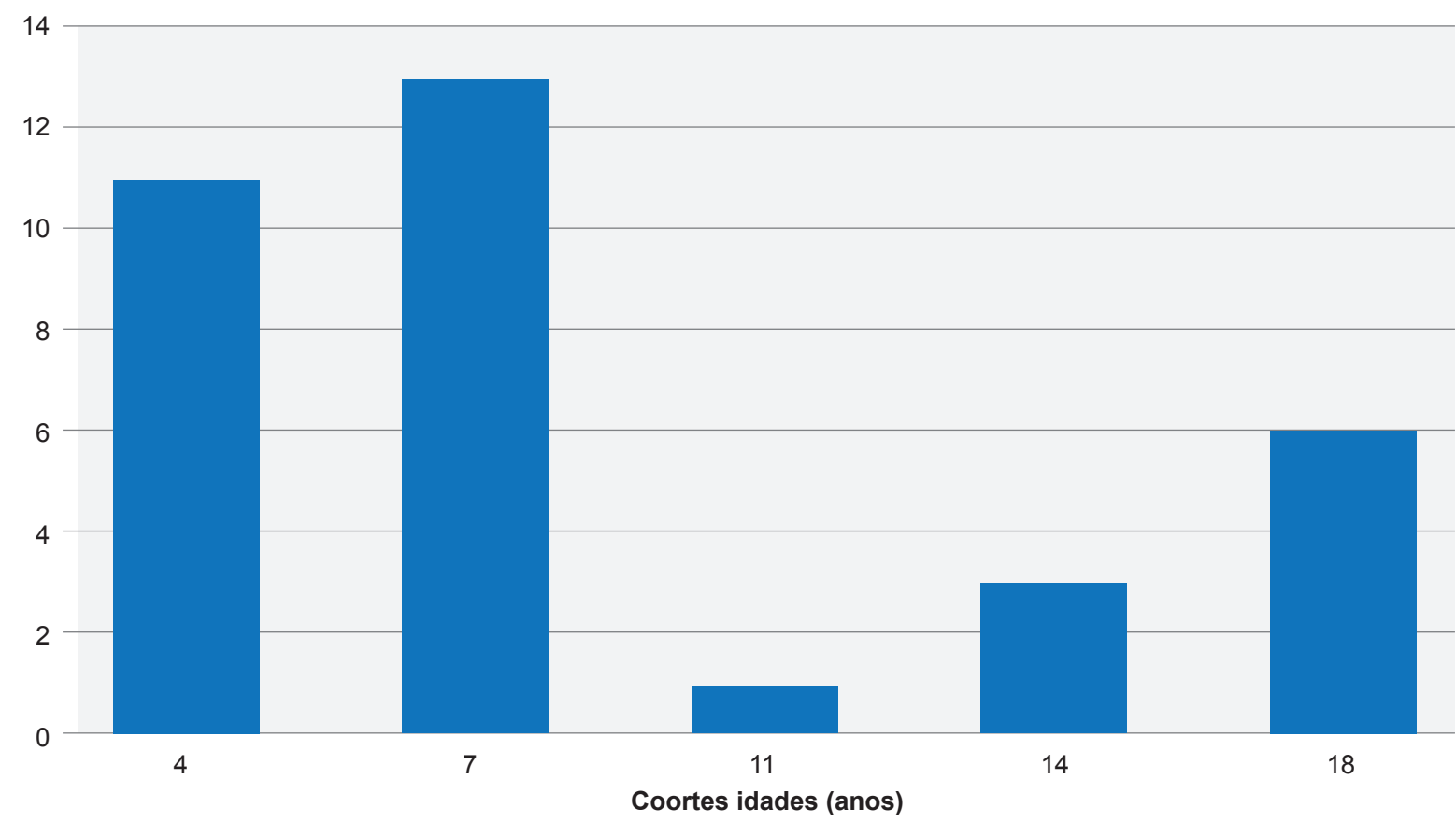

Figura 2 - Histograma de frequência. Representação gráfica da frequência das infeções urinárias causadas por bactérias produtoras de $\beta$-lactamases e adquiridas na comunidade distribuída por coortes de idades 
Amoxicilina + ácido clavulânico $\left(17^{\star}\right)$

Ciprofloxacina $\left(32^{*}\right)$

Meropenem/ Imipenem $\left(34^{*}\right)$

Gentamicina $\left(34^{*}\right)$

Nitrofurantoína $\left(23^{*}\right)$

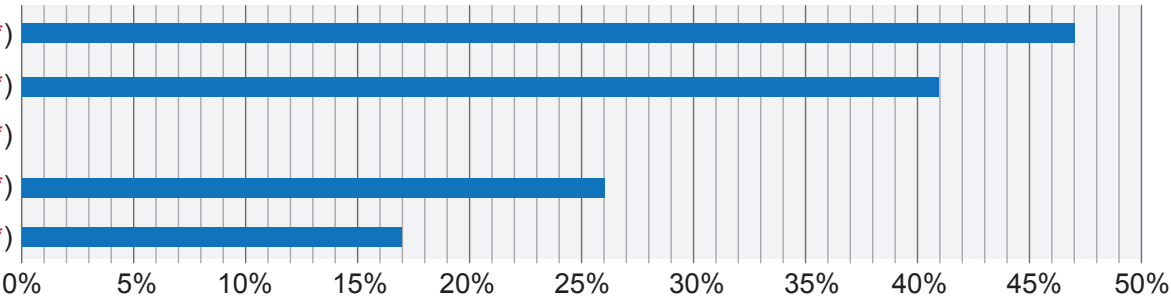

Figura 3 - Percentagem de bactérias produtoras de $\beta$-lactamases resistentes aos antibióticos testados

* O número de estirpes testadas para cada antibiótico é apresentado entre parêntesis

imipenem) e nove (26\%) eram resistentes à gentamicina. Nos 17 casos em que o antibiograma incluiu a amoxicilina/ácido clavulânico, oito (47\%) eram resistentes a este antibiótico. Em relação à ciprofloxacina, 13 (41\%) das 32 amostras testadas eram-Ihe resistentes.

\section{Tratamento e evolução clínica das IU causadas por bactérias produtoras de ESBL-AC}

A terapêutica empírica foi realizada com cefuroxime-axetil em 19 (56\%) casos e com amoxicilina/ácido clavulânico em 15 (44\%), de acordo com o protocolo de orientação clínica em vigor no Hospital Pediátrico-CHUC.

Foram reavaliadas $33(97 \%)$ crianças: $30(91 \%)$ estavam assintomáticas e três (9\%) mantinham queixas; $24 / 33$ $(73 \%)$ repetiram urocultura, que foi positiva em três (13\%). A evolução clínica e o resultado da urocultura de controlo apresentam-se na Fig. 4. Nos três casos sintomáticos, dois tinham urocultura de controlo positiva e, dos 21 assintomáticos que fizeram controlo, apenas um tinha urocultura positiva.

Como apresentado na Tabela 1, houve três casos em que a urocultura de controlo foi positiva, estando dois sintomáticos e um assintomático. Todos tinham o diagnóstico de pielonefrite e estavam sob tratamento com cefuroxime-axetil. Foi alterada a antibioterapia nos dois sintomáticos: num para cotrimoxazol e noutro para carbapenemo (meropenem). Em todos estes doentes a segunda urocultura de controlo revelou-se negativa. Todos estavam assintomáticos na segunda reavaliação.

Houve quatro crianças com urocultura de controlo ne-

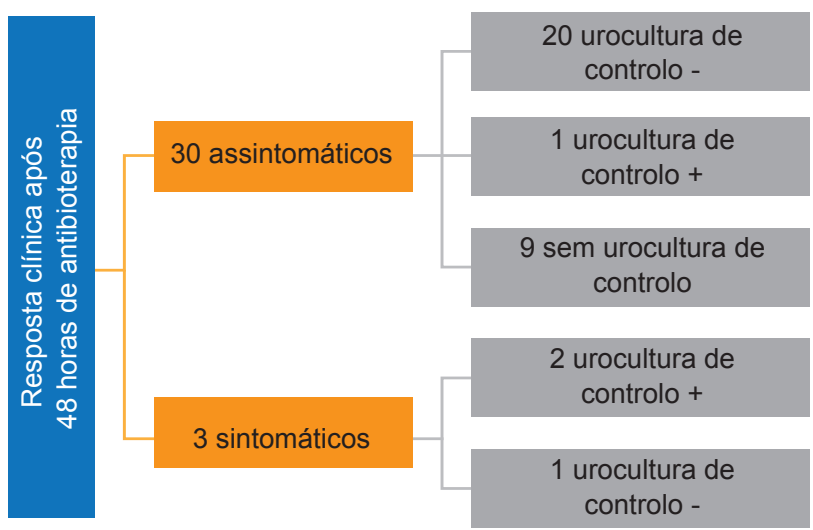

gativa nas quais foi modificada a antibioterapia empírica inicial após conhecimento do antibiograma: num dos casos foi alterada para um carbapenemo por agravamento clínico, e nos restantes três casos foi prescrito cotrimoxazol, apesar de estarem assintomáticos.

Dos 28 casos que foram tratados apenas com antibióti$\cos \beta$-lactâmicos, 27 (96\%) foram sujeitos a reavaliação e todos tiveram resolução dos sintomas.

Houve a necessidade de internamento em três casos, com uma duração média de 4,3 dias (2-9 dias), todos pequenos lactentes.

Em 30 (88\%) casos foi realizada investigação imagiológica, não se tendo detetado malformações nefro-urológicas.

Nos seis meses seguintes, houve nova IU em 4/34 $(12 \%)$ casos, ocorrendo em todos nos primeiros 30 dias após a primeira IU, tal como apresentado na Tabela 2. Em todas estas crianças o tratamento tinha sido com antibióti$\cos \beta$-lactâmicos e tinha havido cura clínica e laboratorial após o primeiro episódio. Foram identificados microrganismos não produtores de ESBL em três destes casos, e num caso com pielonefrite aguda foi isolada $E$. coli produtora de ESBL. Os diagnósticos do novo episódio de infeção foram os mesmos do primeiro: três casos de cistite aguda e uma pielonefrite aguda.

\section{DISCUSSÃO}

Este estudo apresenta a experiência de um centro pediátrico no tratamento de IU causadas por bactérias produtoras de ESBL-AC, num período temporal de 10,5 anos.

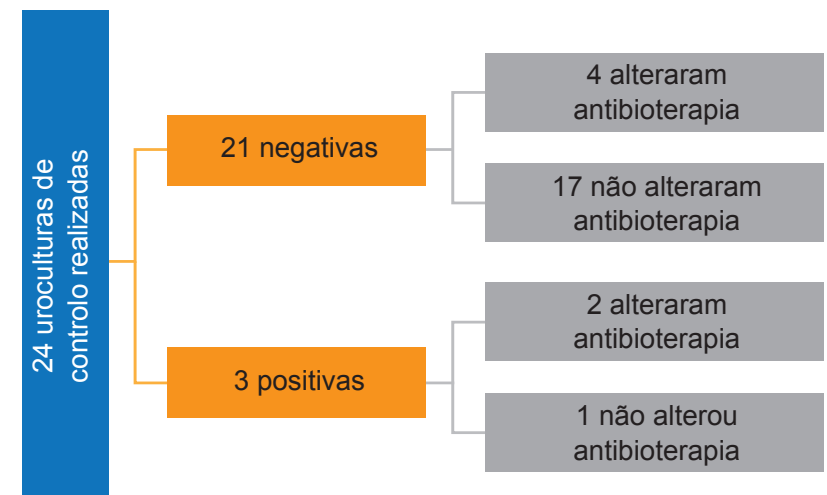

Figura 4 - Evolução clínica e resultado da urocultura de controlo 48 horas após instituição da antibioterapia empírica 
Tabela 1 - Terapêutica antibiótica e evolução dos casos em que a urocultura de controlo foi positiva

\begin{tabular}{|c|c|c|c|c|}
\hline $\begin{array}{l}\text { Bactéria isolada na } \\
\text { primeira infeção urinária }\end{array}$ & $\begin{array}{c}\text { Antibiótico empírico } \\
\text { inicial }\end{array}$ & $\begin{array}{l}\text { Primeira urocultura } \\
\text { de controlo }\end{array}$ & Antibiótico administrado & $\begin{array}{l}\text { Segunda urocultura } \\
\text { de controlo }\end{array}$ \\
\hline Klebsiella pneumoniae & Cefuroxime-axetil & K. pneumoniae & Meropenem & Negativa \\
\hline Escherichia coli & Cefuroxime-axetil & E. coli & Cotrimoxazol & Negativa \\
\hline Escherichia coli & Cefuroxime-axetil & E. coli & $\begin{array}{l}\text { Sem alteração da } \\
\text { antibioterapia }\end{array}$ & Negativa \\
\hline
\end{tabular}

Tabela 2 - Características clínicas e microbiológicas das crianças que repetiram infeção urinária nos seis meses após o primeiro episódio

\begin{tabular}{|c|c|c|c|c|c|c|}
\hline $\begin{array}{l}\text { Tipo de infeção } \\
\text { urinária }\end{array}$ & $\begin{array}{l}\text { Idade } \\
\text { (anos) }\end{array}$ & $\begin{array}{l}\text { Bactéria ESBL } \\
\text { na primeira } \\
\text { infeção } \\
\text { urinária }\end{array}$ & $\begin{array}{c}\text { Antibiótico } \\
\text { inicial na } \\
\text { primeira } \\
\text { infeção urinária }\end{array}$ & $\begin{array}{c}\text { Alteração do } \\
\text { antibiótico na } \\
\text { primeira infeção } \\
\text { urinária }\end{array}$ & $\begin{array}{c}\text { Bactéria na } \\
\text { segunda infeção } \\
\text { urinária }\end{array}$ & $\begin{array}{c}\text { Malformações } \\
\text { nefro-urológicas }\end{array}$ \\
\hline Cistite & 3 & $\begin{array}{l}\text { Proteus } \\
\text { mirabilis }\end{array}$ & Amoxiclav & Não & $\begin{array}{c}\text { Klebsiella } \\
\text { pneumoniae ESBL } \\
\text { negativa }\end{array}$ & Não \\
\hline Cistite & 13 & Escherichia coli & Amoxiclav & Não & $\begin{array}{l}\text { Escherichia coli } \\
\text { ESBL negativa }\end{array}$ & $\begin{array}{l}\text { Sem avaliação } \\
\text { imagiológica }\end{array}$ \\
\hline Cistite & 3 & $\begin{array}{l}\text { Proteus } \\
\text { mirabilis }\end{array}$ & Amoxiclav & Não & $\begin{array}{l}\text { Proteus mirabilis } \\
\text { ESBL negativa }\end{array}$ & $\begin{array}{c}\text { Sem avaliação } \\
\text { imagiológica }\end{array}$ \\
\hline Pielonefrite & 15 & Escherichia coli & $\begin{array}{l}\text { Cefuroxime- } \\
\text { axetil }\end{array}$ & Não & $\begin{array}{l}\text { Escherichia coli } \\
\text { ESBL positiva }\end{array}$ & Não \\
\hline
\end{tabular}

ESBL: ß-lactamases de espectro expandido

Estas infeções foram detetadas pela primeira vez no Hospital Pediátrico-CHUC em 2010 e o seu número manteve-se relativamente estável ao longo do período de estudo. A maioria das crianças foi tratada com antibióticos não carbapenemos $\beta$-lactâmicos, tendo-se observado uma elevada taxa de cura clínica e microbiológica e baixa taxa de recorrência de infeções pelo mesmo agente.

Nos últimos anos, tem-se assistido a um aumento preocupante da identificação de bactérias produtoras de ESBL, não só em infeções associadas aos cuidados de saúde, mas também em infeções AC. Em idade pediátrica, a incidência de IU causadas por estas bactérias tem vindo a aumentar, sendo uma fonte de preocupação no que diz respeito à antibioterapia. ${ }^{25}$

$\mathrm{Na}$ Europa, estas infeções são mais frequentes a sul e oriente. Segundo o Annual Report of the European Antimicrobial Resistance Surveillance Network (EARS-Net) publicado em 2017, a prevalência de $E$. coli resistente às cefalosporinas de terceira geração em Portugal, no triénio 2013 - 2016, foi de $16,1 \%$, estando acima da média europeia $(12,4 \%)$ e tendo sofrido um aumento de $0,4 \%$ entre 2014 e 2016 . No que diz respeito às estirpes invasivas de $K$. pneumoniae, a percentagem de resistências em Portugal foi de $46,7 \%$, sendo a média europeia de $25,7 \% .^{12}$ Estes dados incluem estirpes de adultos e crianças, associadas aos cuidados de saúde e AC.

Num estudo caso-controlo realizado no Hospital Pediátrico - CHUC no período de 2007 a 2009, que procurou determinar fatores de risco e avaliar as infeções causadas por bacilos produtores de ESBL associado aos cuidados de saúde e AC, 39,1\% ( $n=9)$ corresponderam a IU, a maioria AC $(n=6) .{ }^{25}$ Num estudo efetuado em Espanha, no período de 2015 a 2016, em crianças com idade inferior ou igual a 14 anos, a prevalência das IU causadas por E. coli produ- toras de ESBL-AC foi de 9,2\% ( $n=21 / 229) .{ }^{31}$ Num hospital pediátrico em Israel, no período de 2003 a 2010, a prevalência de $E$. coli produtoras de ESBL foi de $1 \%(n=6 / 562)$ e de Klebsiella spp de 0,4\% $(n=2 / 562) .{ }^{32}$

No presente estudo, não se avaliou a prevalência das IU causadas por microrganismos produtores de ESBL, mas observámos que $19 \%(n=34)$ das IU causadas por microrganismos produtores de ESBL foram AC. Neste grupo, o fenótipo ESBL foi mais vezes identificado entre estirpes de E. coli, tal como referido noutros estudos. ${ }^{32}$

Têm sido descritos, em adultos, fatores de risco para colonização por microrganismos produtores de ESBL, assim como fatores de risco associados a IU-AC causadas por estas bactérias. Os dados em idade pediátrica são escassos. No estudo caso-controlo efetuado neste hospital e acima mencionado, foram identificados como fatores de risco a realização de antibioterapia nos 30 dias anteriores e a existência de patologia crónica que obrigasse a múltiplas visitas hospitalares. A ventilação invasiva nos 30 dias anteriores mostrou-se um fator de risco independente, provavelmente por aumentar a colonização por estes microrganismos. ${ }^{25}$ Num estudo caso-controlo realizado na Turquia, no período de 2004 a 2006, que incluiu crianças com IU causada por estas bactérias, verificou-se também que a presença de doença crónica e internamento nos últimos três meses correspondiam a fatores de risco independentes para estas infeções. ${ }^{33}$ Outros fatores de risco descritos são as infeções urinárias recorrentes, as malformações nefro-urológicas, a profilaxia antibiótica com cefalosporinas e as IU prévias por estirpes de Klebsiella spp, mesmo que se tratassem de estirpes não produtoras de ESBL. ${ }^{26}$

As IU em idade pediátrica estão associadas a maior morbilidade não só relacionada com o episódio agudo, assim como a longo-prazo, com o risco de desenvolvimento 
de hipertensão arterial e doença renal crónica. ${ }^{34}$ As cicatrizes renais ocorrem numa pequena proporção das crianças, no entanto, são a causa mais importante das complicações. ${ }^{35} \mathrm{Um}$ estudo de coorte retrospetivo, que englobou uma amostra de crianças dos dois meses aos seis anos com um primeiro ou segundo episódio de IU, obtida de dois estudos longitudinais conduzidos previamente nos Estados Unidos da América, e com uma duração de seguimento de dois anos, encontrou uma relação entre o atraso no início da antibioterapia em crianças com IU febril e o desenvolvimento de cicatriz renal. ${ }^{36}$

Neste estudo, o resultado do antibiograma dos bacilos isolados encontra-se em consonância com os dados da literatura relativamente ao leque restrito de opções terapêuticas. Apesar da resistência aos carbapenemos descrita em diversos estudos, ${ }^{37,38}$ verificámos que todas as estirpes isoladas Ihes eram suscetíveis.

Os antibióticos carbapenemos são a terapêutica de primeira linha recomendada para tratamento das IU causadas por gérmenes produtores de ESBL. No entanto, o seu uso deve ser criterioso pelo aumento da incidência das Enterobacteriaceae resistentes aos carbapenemos em todo o mundo. ${ }^{39}$ Uma explicação para o aparecimento de carbapenemases é o facto da utilização de carbapenemos induzir mutações a nível de proteínas da membrana externa bacteriana, levando também à seleção de $\beta$-lactamases capazes de os hidrolisar. ${ }^{37} \mathrm{~A}$ exposição prévia a antibióticos, nomeadamente cefalosporinas, parece ser um fator de risco independente e consistente para a emergência de bactérias produtoras de ESBL e de carbapenemases. ${ }^{38}$

Antimicrobianos não-carbapenemos têm sido utilizados com sucesso no tratamento destas infeções em adultos, o que tem implicações importantes na prática clínica diária. Alguns autores apontam defesas locais, para além da elevada concentração atingida no sistema urinário para alguns antibióticos que são excretados na urina, como os principais facilitadores de uma evolução favorável nessas situações..$^{40,41}$ No entanto, há poucos estudos em idade pediátrica sobre a utilização destes antibióticos no tratamento das IU causadas por bactérias produtoras de ESBL.

A concentração inibitória mínima (CIM) é um dos parâmetros farmacodinâmicos utilizados como indicadores na escolha do antibiótico, porém é um parâmetro in vitro. A avaliação da resposta clínica deve também ser utilizada nas decisões terapêuticas após utilização do antibiótico in vivo. ${ }^{41}$

Num estudo retrospetivo conduzido num hospital terciário da Finlândia, no período de 2007 a 2016, que englobou 34 crianças e adolescentes até aos 18 anos com IU causada por bactérias produtoras de ESBL, observou-se que $88 \%(n=30)$ destas infeções foram tratadas com sucesso com cefalosporinas. ${ }^{42}$

Adicionalmente, outro estudo retrospetivo realizado em Tóquio entre 2006 e 2016, que incluiu crianças com IU febril causadas por $E$. coli produtoras de ESBL, concluiu que $80 \%$ destas $(n=12 / 15)$ foram tratadas com sucesso com antibióticos não-carbapenemos. ${ }^{41}$
Recentemente, outros antibióticos não-carbapenemos tais como a fosfomicina, têm sido utilizados com sucesso no tratamento das IU causadas por bactérias produtoras de ESBL em adultos. Uma revisão sistemática publicada em 2010 , concluiu que as cistites causadas por estas estirpes apresentaram uma boa resposta clínica à terapêutica com fosfomicina $(93,8 \%, n=75 / 80)$, nomeadamente em estirpes de E. coli. ${ }^{43}$ Apesar da evidência clínica ainda ser limitada, este antibiótico poderá também ser uma opção terapêutica nas IU causadas por estes microrganismos.

Os aminoglicosídeos têm demonstrado eficácia variável, sendo o mais promissor a amicacina. ${ }^{44}$ Um estudo retrospetivo realizado de 2015 a 2016, que incluiu crianças dos dois aos 18 anos com cistites causadas por estirpes de E. coli produtoras de ESBL-AC, concluiu que a cura clínica ocorreu em $96 \%$ ( $n=51 / 53)$ dos casos. ${ }^{45}$

Uma análise retrospetiva realizada na Coreia do Sul, verificou que não existiam diferenças estatisticamente significativas entre as crianças com IU por Enterobacteriaceae produtoras de ESBL tratadas com antibióticos não-carbapenemos (cefotaxima, piperacilina-tazobactan e amicacina) e com carbapenemos. ${ }^{40}$

No nosso estudo, a cura clínica ocorreu em 91\% (n = 30/33) dos casos com terapêutica empírica inicial com cefuroxime-axetil (56\%) ou amoxicilina/ácido clavulânico (46\%). Destes, nos que realizaram urocultura de controlo, a cura laboratorial foi de $95 \%(n=20 / 21)$. Em um caso, a antibioterapia empírica não foi alterada após conhecimento de ausência de suscetibilidade in vitro dado o curso clínico favorável.

A maioria das crianças realizou avaliação imagiológica complementar com ecografia renovesical e não foram detetadas malformações nefro-urológicas.

Neste estudo, quatro crianças tiveram um novo episódio de IU nos seis meses subsequentes ao primeiro episódio. Em um dos casos, a bactéria responsável pela nova infeção correspondia novamente a uma estirpe de $E$. coli produtora de ESBL. Na primeira infeção não tinha sido alterada a antibioterapia empírica, por haver uma resposta clínica favorável.

A informação sobre a taxa de recorrência destas infeções, de acordo com a terapêutica antimicrobiana utilizada, é muito escassa. Um estudo retrospetivo realizado na Coreia do Sul, que comparou os resultados de IU causadas por bactérias produtoras de ESBL tratadas com antibióticos não-carbapenemos e carbapenemos num período de cinco anos, não encontrou diferença estatisticamente significativa na recorrência de IU entre os dois grupos. ${ }^{40}$

Como aspetos fortes deste estudo, destacamos o facto de só ter incluído casos de IU com colheita assética de urina e o ter ocorrido reavaliação clínica em quase todos os casos. Como limitações, destacamos o facto se basear numa análise retrospetiva, com uma amostra relativamente pequena. Adicionalmente, nem todas as crianças fizeram urocultura de controlo.

Tendo em conta os resultados aqui apresentados, as resistências locais, e o conhecimento científico atual, 
parece-nos adequado manter como orientação o tratamento empírico inicial com antibióticos $\beta$-lactâmicos, fazendo um controlo clínico e laboratorial nos casos em que se identifica um microrganismo resistente in vitro ao antibiótico que está a ser utilizado. Se houver cura clínica e laboratorial esse antibiótico poderá ser mantido.

É fundamental manter vigilância microbiológica destas bactérias e, como proposta para estudos futuros, seria importante englobar outros hospitais do país e estendê-lo a crianças com fatores de risco. Seria também importante conhecer a suscetibilidade à fosfomicina, que foi testada num número reduzido de casos, já que este antimicrobiano pode ser uma opção viável no tratamento das cistites por estes microrganismos.

\section{CONCLUSÃO}

Na última década, cerca de $20 \%$ das IU causadas por Enterobacteriaceae produtoras de ESBL foram adquiridas na comunidade, em doentes sem malformações nefro-urológicas, sem tendência de aumento ao longo dos anos. Embora os números sejam pequenos, a evolução clínica e microbiológica mostrou que a maioria foi tratada com sucesso com antimicrobianos não carbapenemos do grupo

\section{REFERÊNCIAS}

1. Balighian E, Burke M. Urinary tract infection in children. Pedsinreview. 2018;39:3-12.

2. Lagree M, Bontemps S, Dessein R, Angoulvant F, Madhi F, Martinot A, et al. Extended-spectrum $\beta$-lactamase-producing Enterobacteriaceae, national study of antimicrobial treatment for pediatric urinary tract infection. Med Mal Infect. 2018;48:193-201.

3. Oliveira G, Saraiva J. Lições de pediatria Vol. I e II. Coimbra: Imprensa da Universidade de Coimbra; 2017.

4. Jacoby GA. Extended-spectrum beta-lactamases and other enzymes providing resistance to oxyimino-beta-lactams. Infect Dis Clin North Am. 1997;11:875-87.

5. Rodríguez-Baño J, Alcalá JC, Cisneros JM, Grill F, Oliver A, Horcajada JP, et al. Community infections caused by extended-spectrum- $\beta$ lactamase-producing Escherichia coli. Arch Intern Med. 2008;168:1897902.

6. Cantón R, Novais A, Valverde A, Machado E, Peixe L, Baquero F, et al. Prevalence and spread of extended-spectrum- $\beta$-lactamase-producing Enterobacteriaceae in Europe. Clin Microbiol Infect. 2008;14:S144-53.

7. Calbo E, Romaní V, Xercavins M, Gómez L, Vidal CG, Quintana S, et al. Risk factors for community-onset urinary tract infections due to Escherichia coli harbouring extended-spectrum beta-lactamases. J Antimicrob Chemother. 2006;57:780-78.

8. Lukac PJ, Bonomo RA, Logan LK. Extended-spectrum $\beta$-lactamaseproducing Enterobacteriaceae in children: old foe, emerging threat. Clin Infect Dis. 2015;60:1389-97.

9. Raymond J, Nordmann P, Doit C, Vu Thien H, Guibert M, Ferroni A, et al. Multidrug-resistant bacteria in hospitalized children: a 5-year multicenter study. Pediatrics. 2007;119:e798-803.

10. Park SY, Kang Cl, Wi YM, Chung DR, Peck KR, Lee NY, et al. Risk factors and molecular epidemiology of community-onset, multidrug resistance extended-spectrum $\beta$-lactamase-producing. Korean J Intern Med. 2017;32:146-57.

11. Flokas ME, Detsis M, Alevizakos M, Mylonakis E. Prevalence of ESBLproducing Enterobacteriaceae in paediatric urinary tract infections: a systematic review and meta-analysis. J Infect. 2016;73:547-57.

12. European Centre for Disease Prevention and Control. Antimicrobial Resistance Surveillance in Europe 2016. Annual Report of the European Antimicrobial Resistance Surveillance Network (EARS-Net). [consultado 2018 nov 5]. Disponível em: https://ecdc.europa.eu/en/publicationsdata/antimicrobial-resistance-surveillance-europe-2015. dos $\beta$-lactâmicos, com baixa ocorrência de novos episódios de IU nos seis meses seguintes.

\section{PROTEÇÃO DE PESSOAS E ANIMAIS}

Os autores declaram que os procedimentos seguidos estavam de acordo com os regulamentos estabelecidos pelos responsáveis da Comissão de Investigação Clínica e Ética e de acordo com a Declaração de Helsínquia da Associação Médica Mundial.

\section{CONFIDENCIALIDADE DOS DADOS}

Os autores declaram ter seguido os protocolos do seu centro de trabalho acerca da publicação de dados.

\section{CONFLITOS DE INTERESSE}

Os autores declaram não ter conflitos de interesses relacionados com o presente trabalho.

\section{FONTES DE FINANCIAMENTO}

Este trabalho não recebeu qualquer tipo de suporte financeiro de nenhuma entidade no domínio público ou privado.

13. Logan LK, Braykov NP, Weinstein RA, Laxminarayan R; CDC Epicenters Prevention Program. Extended-spectrum $\beta$-lactamase-producing and third-generation cephalosporin-resistant Enterobacteriaceae in children: trends in the United States, 1999-2011. J Pediatric Infect Dis Soc. 2014;3:320-8.

14. Martin D, Fougnot S, Grobost F, Thibaut-Jovelin S, Ballereau F, Gueudet $\mathrm{T}$, et al. Prevalence of extended-spectrum beta-lactamase producing Escherichia coli in community-onset urinary tract infections in France in 2013. J Infect. 2016;72:201-6.

15. Leistner R, Schröder C, Geffers C, Breier AC, Gastmeier P, Behnke M. Regional distribution of nosocomial infections due to ESBL-positive Enterobacteriaceae in Germany: data from the German National Reference Center for the Surveillance of Nosocomial Infections (KISS). Clin Microbiol Infect. 2015;21:255.e1-5.

16. Nieminen $\mathrm{O}$, Korppi M, Helminen M. Healthcare costs doubled when children had urinary tract infections caused by extended-spectrum $\beta$-lactamase-producing bacteria. Acta Paediatr. 2017;106:327-33.

17. Flokas ME, Karanika S, Alevizakos M, Mylonakis E. Prevalence of ESBL-producing enterobacteriaceae in pediatric bloodstream infections: a systematic review and meta-analysis. PLoS One. 2017;12:e0171216.

18. Blomberg B, Jureen R, Manji KP, Tamim BS, Mwakagile DS, Urassa WK, et al. High rate of fatal cases of pediatric septicemia caused by gram-negative bacteria with extended-spectrum beta-lactamases in Dar es Salaam, Tanzania. J Clin Microbiol. 2005;43:745-9.

19. Apisarnthanarak A, Hsu LY, Khawcharoenporn T, Mundy LM. Carbapenem-resistant Gram-negative bacteria: how to prioritize infection prevention and control interventions in resource-limited settings? Expert Rev Anti Infect Ther. 2013;11:147-57.

20. Aris $P$, Boroumand MA, Rahbar M, Douraghi $M$. The activity of fosfomycin against extended-spectrum beta-lactamase-producing isolates of enterobacteriaceae recovered from urinary tract infections: A single-center study over a period of 12 years. Microb Drug Resist. 2018;24:607-12.

21. Liu HY, Lin HC, Lin YC, Yu SH, Wu WH, Lee YJ. Antimicrobial susceptibilities of urinary extended-spectrum beta-lactamaseproducing Escherichia coli and Klebsiella pneumoniae to fosfomycin and nitrofurantoin in a teaching hospital in Taiwan. J Microbiol Immunol Infect. 2011;44:364-8.

22. Doi A, Shimada T, Harada S, Iwata K, Kamiya T. The efficacy of cefmetazole against pyelonephritis caused by extended-spectrum 
beta-lactamase-producing Enterobacteriaceae. Int $\mathrm{J}$ Infect Dis. 2013;17:e159-6.

23. Asakura T, Ikeda M, Nakamura A, Kodera S. Efficacy of empirical therapy with non-carbapenems for urinary tract infections with extendedspectrum beta-lactamase-producing Enterobacteriaceae. Int J Infect Dis. 2014;29:91-5.

24. Álvarez J, Rojas Á, Carvajal C, Revello J, Meza P, Guggiana P, et al. Evaluación de susceptibilidad y respuesta al tratamiento con piperacilina/tazobactam en pacientes con infecciones por Escherichia coli productoras de $\beta$-lactamasas de espectro extendido (BLEE) CTX-M. Rev Chilena Infectol. 2018;35:343-50.

25. Dias A, Oliveira G, Oliveira H, Marques M, Rodrigues F. Bacilos gram negativos produtores de ß-lactamases de espectro expandido num hospital pediátrico. Acta Med Port. 2011;24:S197-206.

26. Dayan N, Dabbah H, Weissman I, Aga I, Even L, Glikman D. Urinary tract infections caused by community-acquired extended-spectrum $\beta$-lactamase-producing and nonproducing bacteria: a comparative study. J Pediatr. 2013;163:1417-21.

27. Haverkate MR, Platteel TN, Fluit AC, Cohen Stuart JW, Leverstein-van Hall MA, Thijsen SF, et al. Quantifying within-household transmission of extended-spectrum $\beta$-lactamase-producing bacteria. Clin Microbiol Infect. 2017;2346.e1-.e7.

28. Herindrainy $P$, Rabenandrasana MA, Andrianirina ZZ, Rakotoarimanana FM, Padget M, de Lauzanne A, et al. Acquisition of extended spectrum beta-lactamase-producing enterobacteriaceae in neonates: a community based cohort in Madagascar. PLoS One. 2018;13:e0193325.

29. Direção-Geral de Saúde. Diagnóstico e tratamento da infeção do trato urinário em ildade pediátrica. Norma n 8, 2012. Lisboa: DGS: 2012.

30. Reaffirmation of AAP Clinical Practice Guideline: The Diagnosis and Management of the Initial Urinary Tract Infection in Febrile Infants and Young Children 2-24 Months of Age. Pediatrics. 2016;138:e20163026.

31. Pérez Heras I, Sanchez-Gomez JC, Beneyto-Martin P, Ruano-de-Pablo L, Losada-Pinedo B. Community-onset extended-spectrum $\beta$-lactamase producing Escherichia coli in urinary tract infections in children from 2015 to 2016: prevalence, risk factors, and resistances. Medicine. 2017;96:e8571.

32. Sakran W, Smolkin V, Odetalla A, Halevy R, Koren A. Communityacquired urinary tract infection in hospitalized children: etiology and antimicrobial resistance. A comparison between first episode and recurrent infection. Clin Pediatr). 2015;54:479-83

33. Topaloglu R, Er I, Dogan BG, Bilginer Y, Ozaltin F, Besbas N, et al. Risk factors in community-acquired urinary tract infections caused by ESBLproducing bacteria in children. Pediatr Nephrol. 2010;25:919-25.
34. Stein R, Dogan HS, Hoebeke P, Kočvara R, Nijman RJ, Radmayr C, et al. Urinary tract infections in children: EAU/ESPU guidelines. Eur Urol. 2015;67:546-58.

35. Westwood ME, Whiting PF, Cooper J, Watt IS, Kleijnen J. Further investigation of confirmed urinary tract infection (UTI) in children under five years: a systematic review. BMC Pediatr. 2005;5:2.

36. Shaikh N, Mattoo TK, Keren R, Ivanova A, Cui G, Moxey-Mims M, et al Early antibiotic treatment for pediatric febrile urinary tract infection and renal scarring. JAMA Pediatr. 2016;170:848-54

37. Tamma PD, Rodriguez-Bano J. The use of noncarbapenem $\beta$-lactams for the treatment of extended-spectrum $\beta$-lactamase infections. Clin Infect Dis. 2017;64:972-80.

38. Marchaim D, Chopra T, Bhargava A, Bogan C, Dhar S, Hayakawa K, et al. Recent exposure to antimicrobials and carbapenem-resistant Enterobacteriaceae: the role of antimicrobial stewardship. Infect Control Hosp Epidemiol. 2012;33:817-30.

39. Han SB, Lee SC, Lee SY, Jeong DC, Kang JH. Aminoglycoside therapy for childhood urinary tract infection due to extended-spectrum $\beta$-lactamase-producing Escherichia coli or Klebsiella pneumoniae. BMC Infect Dis. 2015;15:414.

40. Lee B, Kang SY, Kang HM, Yang NR, Kang HG, Ha IS, et al. Outcome of antimicrobial therapy of pediatric urinary tract infections caused by extended-spectrum $\beta$-lactamase-producing enterobacteriaceae. Infect Chemother. 2013;45:415-21.

41. Abe $Y$, Inan-Erdogan I, Fukuchi $K$, Wakabayashi H, Ogawa $Y$, Hibino $S$, et al. Efficacy of non-carbapenem antibiotics for pediatric patients with first febrile urinary tract infection due to extended-spectrum betalactamase-producing Escherichia coli. J Infect Chemother. 2017;23:51722.

42. Vakkilainen S, Pätäri-Sampo A, Saxén H. Outcome of children with ESBL-E. coli acute pyelonephritis treated with cephalosporins. J Infect. 2018;77:75-81.

43. Falagas ME, Kastoris AC, Kapaskelis AM, Karageorgopoulos DE. Fosfomycin for the treatment of multidrug-resistant, including extendedspectrum beta-lactamase producing, Enterobacteriaceae infections: a systematic review. Lancet Infect Dis. 2010;10:43-50.

44. Madhi F, Jung C, Timsit S, Levy C, Biscardi S, Lorrot M, et al. Febrile urinary-tract infection due to extended-spectrum beta-lactamaseproducing Enterobacteriaceae in children: a French prospective multicenter study. PLoS One. 2018;13:e0190910.

45. Polat M, Kara SS. Once-daily intramuscular amikacin for outpatien treatment of lower urinary tract infections caused by extended-spectrum $\beta$-lactamase-producing. Infect Drug Resist. 2017;10:393-9. 